\title{
EVALUATING ADDITION OF A MEMBRANE LAYER IN VACUUM INFUSION PROCESSING OF FIBER REINFORCED EPOXY COMPOSITES IN TERMS OF FLEXURAL PROPERTIES AND VOID CONTENT
}

\author{
A. Tuğrul SEYHAN ${ }^{1,2, *}$ \\ ${ }^{1}$ Department of Materials Science and Engineering, Faculty of Engineering, Anadolu University, \\ İki Eylül Campus, 26555 Eskişehir, Turkey \\ ${ }^{2}$ Composite Materials Manufacturing Science Laboratory (CMMSL), Research and Application Center of Civil Aviation \\ (RACCA), Anadolu University, İki Eylül Campus, 26555 Eskişehir,Turkey
}

\begin{abstract}
The effect of addition of a membrane layer in vacuum infusion process was investigated with emphasis being placed on the flexural properties and void content of the resulting composites. E-glass fiber and epoxy were, respectively, used as filler and matrix constituents to produce the composites. Conducting a facile TGA based methodology, fiber volume fraction, density, and void content measurements were carried out on the samples taken from different zones across the composite parts. Despite giving rise to the composite thickness, $16 \%$ high compared to the part thickness produced by vacuum infusion without any membrane, infusion with incorporation of the membrane layer resulted in a relatively high composite fiber volume fraction with reduced void content at no expense of compaction. At the vacuum port, flexural strength and modulus values of the composites produced by membrane added vacuum infusion process (MAVIP) were found to be, respectively, 11 and $8 \%$ higher than those of the composites produced by conventional vacuum infusion process (CVIP). On the other hand, at the inlet port, composites produced by MAVIP exhibited 12 and $9 \%$ high flexural strength and modulus values compared to those produced by CVIP. Moreover, the load-deflection response $(\mathrm{P} / \delta)$ of the samples taken from different zones along the parts was correlated with respect to their length-to-width aspect ratio $(\mathrm{L} / \mathrm{b})$ based on the plane strain analytical approach. Once $\mathrm{L} / \mathrm{b}$ aspect ratio was greater than 6.5, plane strain approach was found to converge to more definite beam modulus values, regardless of the process used.
\end{abstract}

Keywords: Composite materials, Mechanical testing, Thermogravimetric analysis (TGA), Mechanical properties

\section{INTRODUCTION}

Conventional vacuum infusion process (CVIP) has been widely used to manufacture large composite parts for various applications, including civil engineering and defense [1-3]. It simply involves applying a fiber preform onto a rigid tool followed by infusion of the preform with a catalyzed liquid resin, and finally the cure of the infused resin under vacuum. In CVIP, the vacuum draws resin in, while simultaneously drawing air out to reduce the incidence of surface porosity and void content in resulting composites [1-3]. However, even though the additional attachments, such as bleeders and breathers, evacuated air is not effectively prevented from being accompanied by the resin used in the process. This leads to resin waste that may clog up vacuum lines, even though the catch pots used for keeping the vacuum pump safe [2]. If contaminated with the catalyzed resin, vacuum lines are either to be flushed with solvent or thrown away in ease. On the other hand, a pressure gradient that develops during infusion process causes a thickness gradient across the part, which adversely influences the fiber volume fraction variation across the part [3-5]. CVIP remains therefore vastly lacking in terms of the required part quality for advanced composite applications. In order to enhance the repeatability of the part characteristics, CVIP has been recently modified with a gas-permeable membrane, a thin, flexible film or fabric typically made from either polytetrafluoroethylene (PTFE) or thermoplastic polyurethane (TPU), that permits uniform vacuum distribution during infusion [3-4]. These types of membranes have been privileged as potential pathways to easy, repeatable, and high-quality vacuum infusion. It was 
recommended that they be used as an additional layer in all or some parts of the fabric preform, thus bringing up a full vacuum gradient combined with a continued degassing across the part surface during process [3-6]. This feature enables MAVIP to be superior over CVIP where the vacuum is applied just at one end-edge of the part. More importantly, dry-spot removal during MAVIP is asserted to be achieved without optimized vent placement, as opposed to CVIP wherein it is inevitable.

Characterization of void content and porosity are important not only in terms of quality assessment of mechanical property integrity in composites, but also in terms of the efficiency of the manufacturing process used for their production. Voids and porosity can occur either at the ply interfaces at interlaminar region or within the individual plies at intralaminar region. Oliver [7] stated that the void formation and growth are primarily owing to entrapped volatiles during the lay up in a humid air or during impregnation of the fiber reinforcement with resin. He also concluded that higher temperatures lead to higher volatile pressure, and that void growth is of vitally significance once the volatile vapor pressure exceeds the hydrostatic resin pressure. Harper et al. [8] revealed that producing composite products with very low void volume contents lies in selecting the most appropriate manufacturing process for the fiber and matrix constituents in hand. This is because when the liquid resin viscosity dramatically increases and gelation takes place, no matter what types of the fiber stacking sequence are used, the voids are inclined to be locked into the resin matrix. They also concluded that the applied pressure on the laminate is not necessarily called a vigorous factor, as the hydrostatic pressure can be low, even if the applied pressure is high, which in turn inevitably leads to void formation and growth. Yu and Middleman [9] concluded that the driving force for void formation in composite processing is associated with the heterogeneous nature of the preform at the micro-structural level, where it is composed of arrays of fiber bundles or tows, which affect their permeability as well. They showed that in fast flow conditions, the resin has a propensity to flow around the tows, as the open space between adjacent tows is much less resistant to flow than the tows themselves. Void formation occurs primarily within the tows in this flow regime. Kurematsu and Koshi [10] concluded that the flow front within the fiber tows may be related to the bulk flow front when capillary forces are more dominant than viscous forces. In this case, void formation may occur in the open space between the tows. Voids are also detrimental to final composite mechanical properties, especially to those which are matrix dominated. Judd and Wright [11] reported that, regardless of resin and fiber types or fiber surface treatment, the interlaminar shear strength of a composite decreased by about $7 \%$, even if $1 \%$ void level exists in final parts. Ghoirse [12] investigated that interlaminar shear and flexural strengths were, respectively, reduced by 10 and $5 \%$ for every $1 \%$ addition of void contamination. Costa et al. [13] reported that interlaminar shear strength (ILSS) values decreased by about $34 \%$ for carbon/epoxy fabric laminates when their void content increased from 0.55 up to $5.60 \%$. Overall speaking, studies concerned with the relationship between the composite mechanical properties and the voids concluded that the void problem cannot be resolved simply by one measure taken [14]. This is because sources of voids can be dependent on many factors, including those that occur when cutting, collating and laying up layers, during which air can become entrapped between the individual plies. In addition to this, air can be entrapped in the resin itself during the mixing and thus serves as nucleation sites during impregnation of the preforms, which in turn becomes highly influential in the quality of the resulting composites [6-14]. To reduce these challenges, MAVIP is believed to have potential to open up a new perspective in advanced composite processing.

The most significant contribution of this paper is to be the first study in the literature that focuses on evaluating the effect of the membrane layer on the flexural properties of the composites on the basis of the fiber volume fraction and void content distribution along the composite parts. CVIP stands as a basis for property comparison purposes. A facile TGA based methodology was conducted to perform composite fiber volume fractions and void content measurements. The load-deflection response of the beams under flexural loading was investigated as a function of the length-to-width aspect ratio. Plane strain analytical approach was then executed to correlate the experimental findings. The results obtained were then discussed in a concise manner with emphasis on the relationships among the flexural properties, fiber volume fraction and void contents of the composite parts produced. 


\section{EXPERIMENTAL}

\subsection{Composite Part Production}

The composite parts were produced from angle ply $( \pm 45)_{2 S}$ E-glass fabric preforms. A sheet of polyurethane based membrane layers, commercially coded as A2049, which has been recently developed by Trans-textile $\mathrm{GmbH}$ for low temperature vacuum infusion applications, was utilized when producing the parts by using MAVIP. Epikote epoxy resin MGS (L-160) and its corresponding aromatic hardener Epicure MGS (H-163) were purchased from HEXION corp. They were mixed at a ratio of $100: 35 \mathrm{w} / \mathrm{w}$ by a mechanical stirrer followed by being degassed for $30 \mathrm{~min}$. The final mixture was then employed as matrix constituent to impregnate the glass fabric preforms. Once the infusion was terminated, the parts were allowed to cure at room temperature followed by post curing at 45 and $80^{\circ} \mathrm{C}$ for 24 and $1 \mathrm{~h}$, respectively.

\subsection{Composite Density, Fiber Volume Fraction and Void Content Measurements}

Composite density measurements were made according to ASTM D-792. The testing procedure requires that the density of the composite samples be measured using Archimedes' principle, where the specimen is first weighed in air and then once again weighed when submerged in water. Mechanical property measurements were carried out afterwards. Fiber and void volume fractions of the each corresponding sample were measured last. A TA Instruments Q-500 Thermogravimetric Analyzer (TGA) was used to more accurately determine the glass fiber content of the composite specimens. For this purpose, the specimens were taken from different zones across the parts. The composites were machined into a tiny cylindrical shape, large enough to fit a TGA pan, to ensure that the maximum heat transfer surface area is provided in order to completely get rid of the remaining matrix resin left at the end of the burn-out process inside the chamber. Small amounts of pure epoxy resin and the composites were separately heated at constant heating rate of $10^{\circ} \mathrm{C} / \mathrm{min}$. under nitrogen atmosphere with a flow rate of $50 \mathrm{ml} / \mathrm{min}$ until observing a relatively flat region of the TGA due to the pyrolysis of the epoxy resin just prior to final drop-off. The inflection point in the TGA of polymer pyrolysis was taken into careful consideration when calculating the fiber weight fractions according to the equation (1). The fiber volume fractions were then calculated from the TGA data obtained for each sample by using the fiber and matrix densities. Void contents were then calculated for specimens subjected to the resin burn-out in the TGA chamber by using the equation (2) where $\rho_{\text {composite }}$ refers to experimentally determined composite densities.

$$
\begin{aligned}
& \% W_{\text {fiber TGA }}=\frac{\left(\text { Residue }_{\text {composite }}-\text { Residue }_{\text {epoxy matrix }}\right)}{\left(100-\text { Residue }_{\text {epoxy matrix }}\right)} \times 100 \\
& V_{v}=\left[1-\rho_{\text {composite }} x\left[\frac{\% W_{\text {matrix }}}{\rho_{\text {matrix }}}+\frac{\% W_{\text {fiber }}}{\rho_{\text {fiber }}}\right]\right] \times 100
\end{aligned}
$$

\subsection{Composite Flexural Modulus and Strength}

Angle-ply preform configuration was intentionally chosen to distinctly differentiate between the process induced effects on the flexural properties of the composites. This is because resin permeability of that preform is very low and prone to void formation, which helps ease the process efficiency comparison. A three-point bend test was also intentionally selected, as the shear stress is supposed to be constant along the length of the beam under this configuration while being directly proportional to the applied force [15-17]. To perform the tests, a three point flexural test fixture was hooked up to the load cell of an Instron Universal Testing Machine (5541) coupled with a calibrated loading cell of $2000 \mathrm{~N}$. Testing was conducted at a crosshead speed of $5 \mathrm{~mm} / \mathrm{min}$ according to ASTM D790-10 standard at a span-todepth ratio of 32 by using the specimens, $25 \mathrm{~mm}$ in width and $80 \mathrm{~mm}$ in length, which were randomly 
taken from the inlet and the vacuum ports of the composite parts. The flexural strength (S) and modulus $\left(\mathrm{E}_{\mathrm{f}}\right)$ values were calculated from the load-deflection data recorded during the testing, as follows.

$$
\begin{gathered}
S=\frac{3 P_{\max } L}{2 b h^{2}}\left\lceil 1+6\left(\frac{D}{L}\right)^{2}-4\left(\frac{h}{L}\right)\left(\frac{D}{L}\right)\right\rceil \\
E_{f}=\frac{m L^{3}}{4 b h^{3}}
\end{gathered}
$$

where $\mathrm{L}, \mathrm{b}$ and $\mathrm{h}$ are the support span, width and depth of the specimen, respectively, $\mathrm{m}$ is the slope of the tangent to the initial straight line portion of the load deflection curve. D and P are, respectively, the maximum deflection and the maximum load prior to failure. On the other hand, the load deflection ratio $(\mathrm{P} / \delta)$ was additionally studied as a function of the length to width ratio $(\mathrm{L} / \mathrm{b})$. Assuming that shear deformations for the samples are negligible, as they are out of plane-loaded and the maximum bending moment takes place at the mid-length of the beam in a three point flatwise flexure, and that the span to depth ratio is large enough, leading to occurrence of compression and tension loads on the top and the bottom surfaces of the beams, respectively, the midspan deflection was proposed to be calculated from the below equation.

$$
\delta=\frac{P L^{3}}{48\left(E_{x}^{f}\right) I_{Y}}
$$

where $E_{x}^{f}$ the effective beam bending modulus and $I_{Y}$ is the moment of inertia as follows for the specimens used in this study.

$$
I_{Y}=\frac{b h^{3}}{12}
$$

For experimental-analytical correlations, the interpretation was enriched by representing the different experimental load deflection ratio $(\mathrm{P} / \delta)$ as a function of $E_{x}$ on a single plot. For this purpose, equations 6 and 7 are combined and rearranged as follows.

$$
\left(\frac{P}{\delta}\right) \frac{L^{2}}{h^{3}}=4 \frac{\left(E_{\chi}^{f}\right)}{\frac{L}{b}}
$$

where $(\mathrm{P} / \delta)$ is experimentally measured values for each sample and the $E_{x}^{f}$ is the effective beam bending modulus values, mathematically derived for symmetrical composite parts according to the below equation.

$$
\left(E_{x}^{f}\right)=\frac{12}{h^{3} D_{11}^{*}}
$$

$D_{11}^{*}$ is a component of the laminate bending compliance matrix. A MATLAB code was developed and used for calculating the matrix components based on the classical laminate theory. By plotting the right hand side of the equation (5) and using the effective beam bending modulus, incessant curves were obtained. Different fiber volume fractions of each mechanically tested sample were considered and their corresponding correlations were made accordingly with respect to the process used. In this manner, flexural samples with the same length, but in different widths $(10,15,20,25 \mathrm{~mm})$ were sectioned from the inlet and vacuum ports of the produced parts. In Figure 1, on the right hand side is the corresponding specimen codes designated to six different zones selected over the composite parts, while on the left hand-side is the schematic illustration of the individual components of the MAVIP with a photo taken during the process underneath it. 


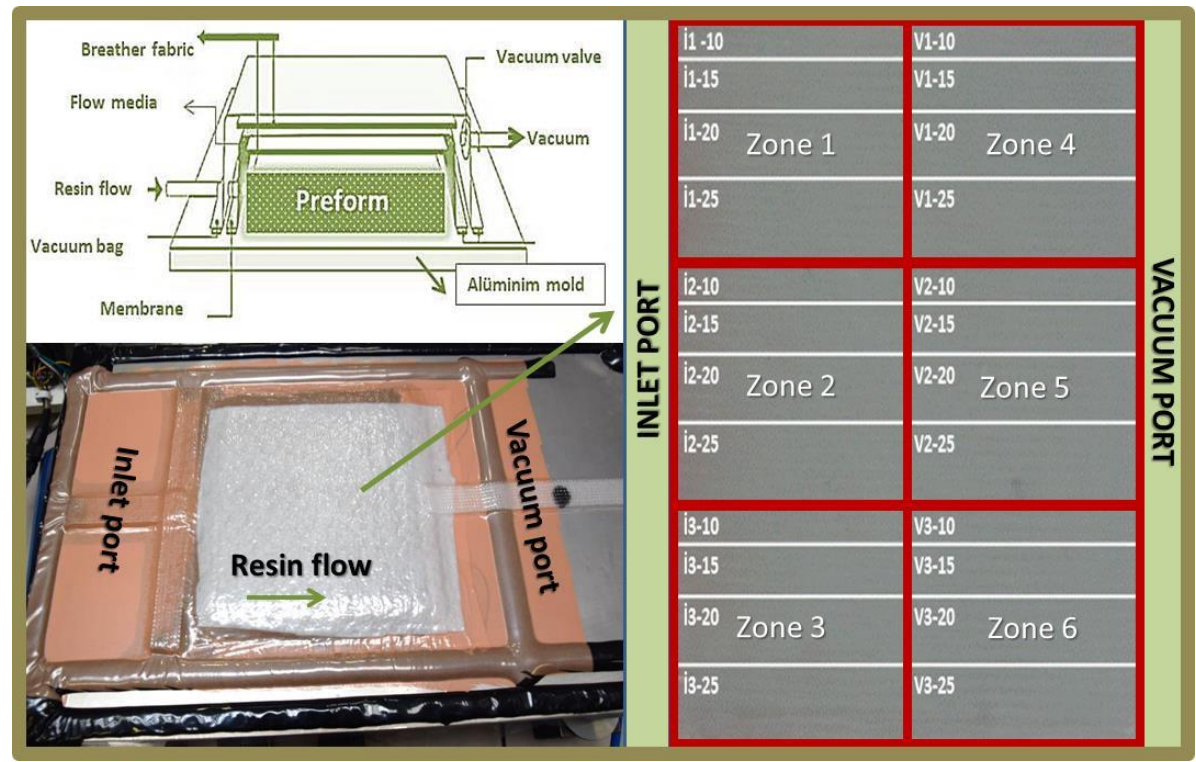

Figure 1. Designation of 3 point bending specimens sectioned from different regions of the composite according to the locations with respect to inlet and vacuum port, where E.g. I2-10 stands for the $10 \mathrm{~mm}$ wide specimen cut from Zone 2.

\section{RESULTS AND DISCUSSION}

Figure $2 \mathrm{a}$ and $\mathrm{b}$ give, respectively, the composite flexural strength and modulus values calculated according to the ASTM procedure described in the experimental part. Composite samples were sectioned from near the inlet and vacuum ports of the parts. Regardless of the ports, the composites produced by MAVIP were found to exhibit to some extent high strength and modulus values relative to the composites produced by CVIP. When each port was evaluated on its own, regardless of the process used, samples possessed higher strength and modulus values at the vacuum port than at the inlet port, as expected.
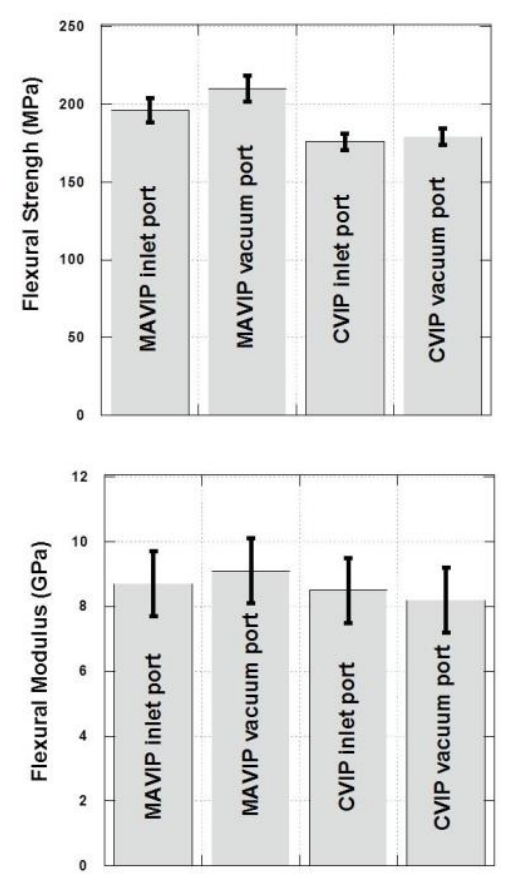

Figure 2 The flexural strength and modulus of the composites with respect to the ports 
At the vacuum port, strength and modulus values of the composites produced by MAVIP are, respectively, 11 and $8 \%$ higher than those of the composites produced by CVIP. On the other hand, at the inlet port, the same trend is also valid such that composites produced by MAVIP revealed 12 and 9 $\%$ high strength and modulus values compared to those produced by CVIP. Generally speaking, the higher fiber volume fraction accompanied by the lower void content is the key to obtaining composites with high mechanical strength values. From this point of view, it is obvious that the thickness and its gradient across the parts, besides different fiber volume fraction variations arising from the own nature of each process play a significant role in the mechanical property findings obtained. Please note that the thickness of the samples, despite slightly, vary depending on the port where they were taken from. Numerically speaking, the overall thickness of the composites produced by MAVIP in this study was found to be about $16 \%$ higher than the overall thickness of composites produced by CVIP. One major reason behind the difference in the results obtained lies in the dynamic part thickness change throughout CVIP because of the pressure gradient that occurs in the zone wetted with the resin, which alters compaction at that zone dependent on time [4-6]. Another reason is that, in CVIP, only closing the resin injection port once the infusion is terminated makes it possible to impose a full and uniform vacuum pressure on the fabric preform, thus enhancing impregnation of the fiber tows while aiding in relocation of the resin from the inlet port through the outlet vent $[18,19]$. As for MAVIP, in a similar manner, uniform vacuum throughout the process is always available and permits continued infusion even after the part is entirely filled with the resin, thus leading to a relatively small thickness gradient accompanied with the insignificant pressure gradient on the part surface. In addition to this, in CVIP, resin bleeding from the vent occurs until the resin is dead, while it is almost continuous during MAVIP. This is why the final thickness taken anywhere throughout the part after cured ends up being higher in MAVIP than in CVIP. By the way, finding the perfect timing to close the inlet port during CVIP is anybody's guess in most cases [5,18]. To sum up, MAVIP holds promise to accomplish the parts with a reduced thickness gradient at no expense of compaction. In other words, it is expected to result in high fiber volume fraction accompanied by low void content along the parts relative to CVIP. By dividing the parts into several zones, as explaneed in the experimental section, it was, by the way, evaluated whether or not MAVIP really provides a more homogenous mechanical property variation across the composite part, as claimed in the light of the hypothetical facts mentioned above in detail. To aid in performance comparison efficiency in between the two processes, density, void and fiber volume fractions of the samples derived from each selected zone were measured using TGA. Figure $3 \mathrm{a}$ and $\mathrm{b}$ is a typical behavior of the samples during TGA measurements. Generally speaking, both the samples, namely, composite and epoxy matrix resin, exhibit two weight loss steps. The first one that occurs at around $200 \mathrm{oC}$ is caused by either the moisture elimination that the samples may most probably have captured when stored on ambient air or by the decomposition of the coupling agent over the glass fiber surface, while the second one indicates the sign of material pyrolysis. Subsequent to the pyrolysis step, both samples left a residue. On the other hand, as seen in the figure, despite to some extent, the behavior of the samples were altered depending on the zone where they were taken from. However, for the samples produced by MAVIP, drop-off lines at the baseline are almost the same independent of the zones, which can be taken as evidence for uniform property characteristics across the parts compared to CVIP. Figure 4 gives the density, fiber volume fraction, and the void content of the composites according to the designated zones, which were produced by CVIP and MAVIP. Please note that zone 1,2 and 3 represent the resin inlet ports, while zones, 4, 5, 6 represent the vacuum port, as already elucidated in Figure 1. No matter what zone is of concern, the highest density, fiber volume fraction, together with the lowest void content were obtained from the composites produced by the MAVIP. These results confirmed the theoretical issues mentioned at the beginning of the text. Moreover, the validity of that trend was also endorsed in the experimental plots highlighted in Figure $5 \mathrm{a}$ and $\mathrm{b}$ where the composites produced by MAVIP exhibited the highest slope value regardless of whether the resin or vacuum ports are of matters. When compared to each other, all the zones nearby the vacuum port have a higher slope than the zones nearby the inlet port, indicating that the strength and modulus values are relatively high at the vacuum port, as expected. This is proportional to the composite flexural property findings obtained according to 
Seyhan / Anadolu Univ. J. of Sci. and Technology A-Appl. Sci. and Eng. 18 (2) - 2017

the ASTM standard such that the higher fiber volume fraction, the lower void content, and thus the higher mechanical properties can be achieved.
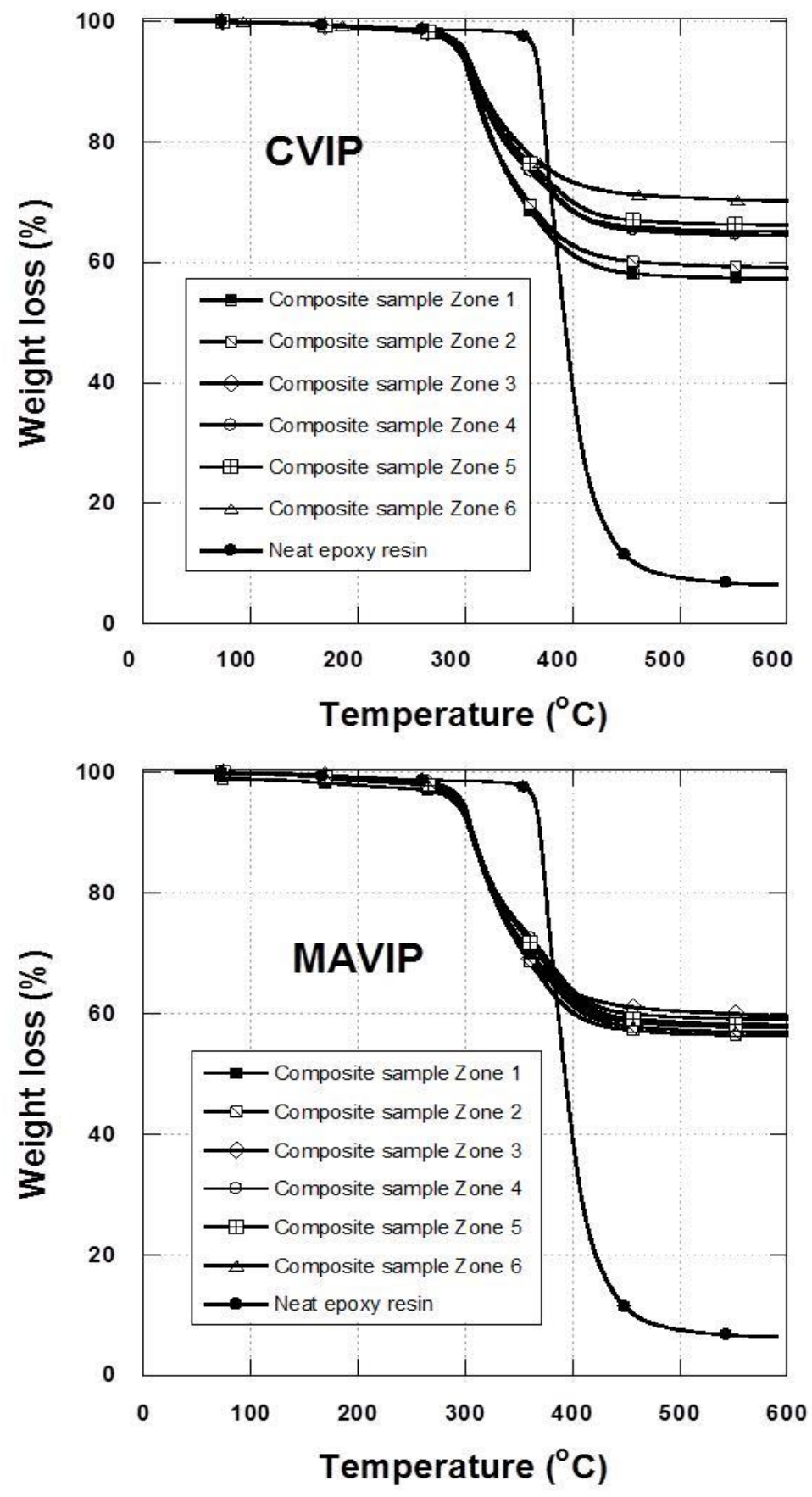

Figure 3. Typical TGA response of the composite samples sectioned from the designated zones over the parts produced by CVIP and by MAVIP 


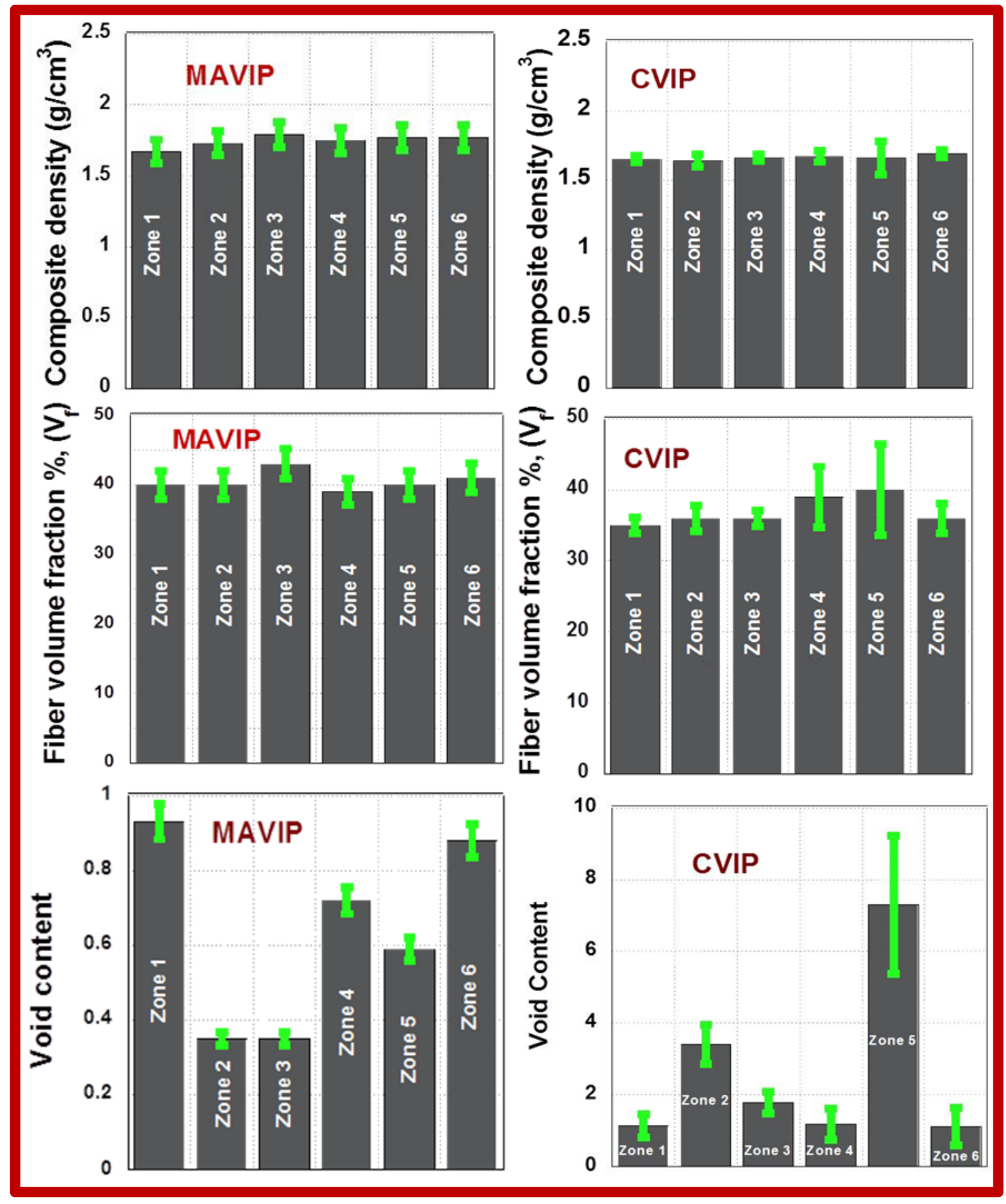

Figure 4. Density, fiber volume fraction, and the void content of the composites with respect to the zones selected 
Seyhan / Anadolu Univ. J. of Sci. and Technology A-Appl. Sci. and Eng. 18 (2) - 2017
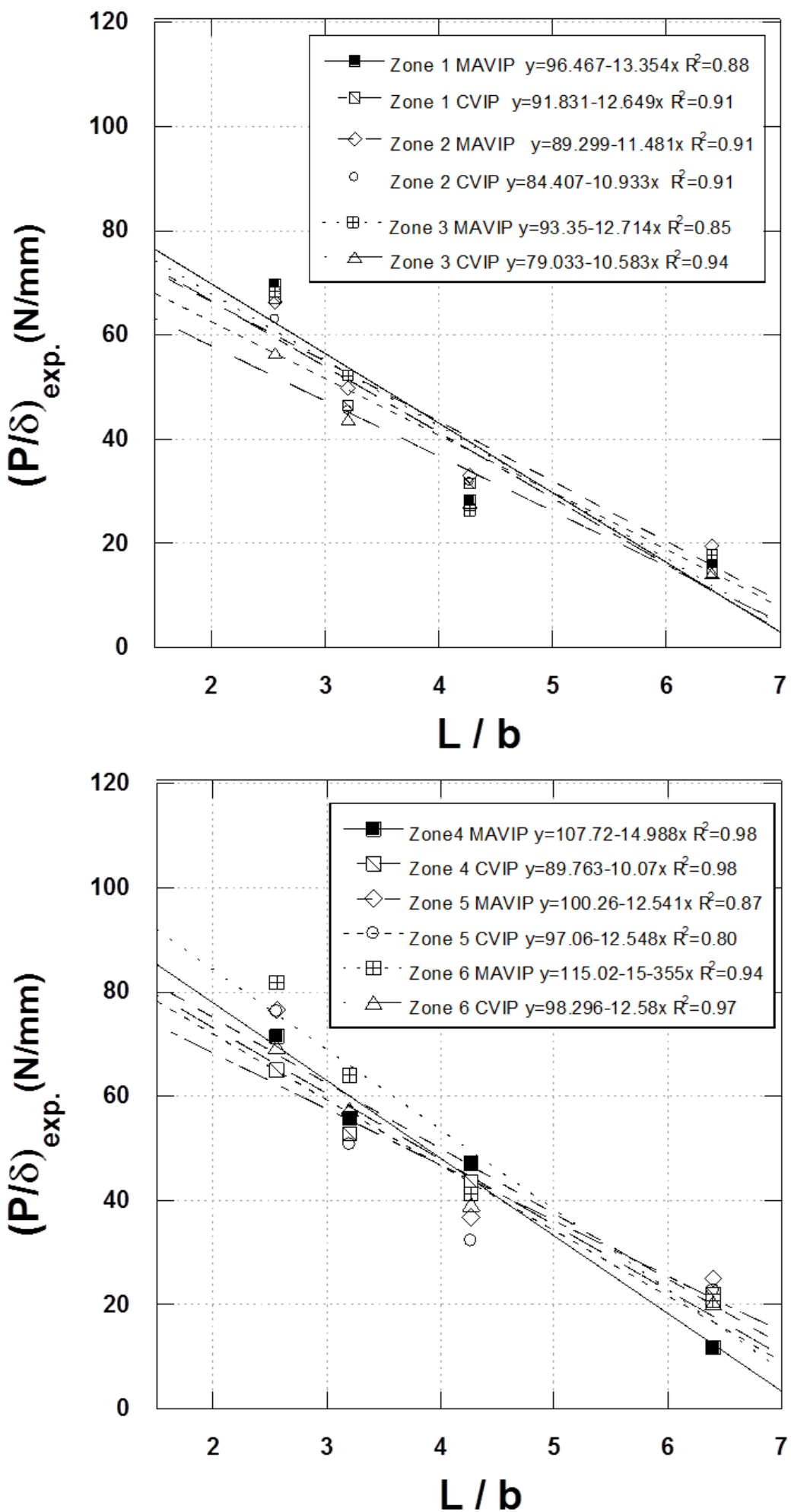

Figure 5. Experimental load deflection values with respect to designated zones in a comparative manner, depending on the distance from the resin and vacuum ports 
Figure 6 gives the correlation of all the experimentally determined modulus values for the composites with the analytical prediction. Note that equation 7 was used to plot the MAVIP and CVIP functions in Figure 6. As seen in the figure, the plane strain approach assumption provides a lower bound for the beam modulus. Moreover, when $\mathrm{L} / \mathrm{b}$ aspect ratio becomes greater than 6.5 , the plane strain approach predicts well the experimental findings, regardless of the process used.

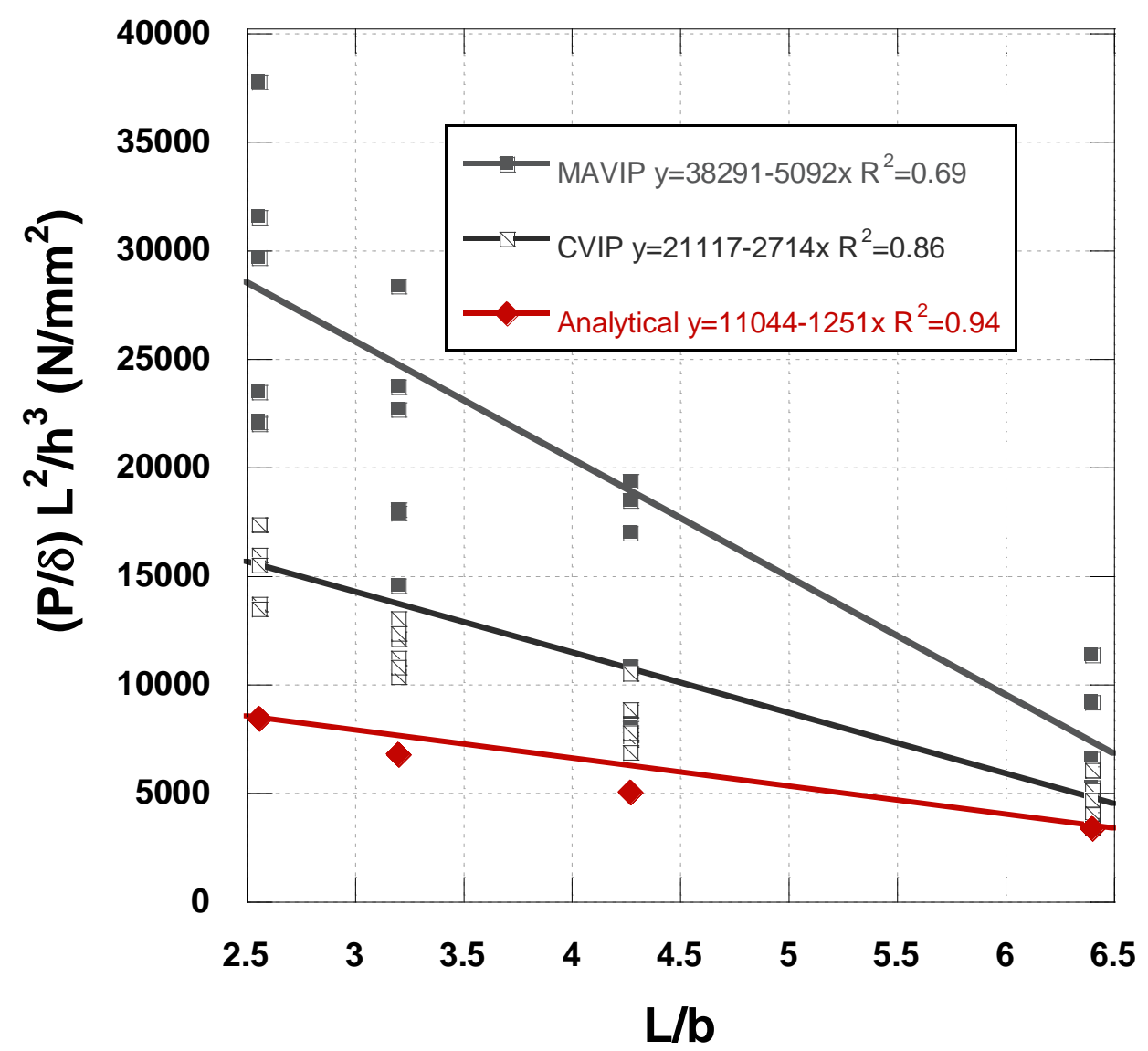

Figure 6 Correlation of the experimental results found with the predictions of the analytical model, taking into account all the zones involved

\section{CONCLUSIONS}

Composite flexural properties were evaluated with emphasis on the effect of the manufacturing process used. Composites were produced by CVIP and MAVIP. Conducting a facile TGA based methodology, density, fiber volume fraction, and void content measurements were carried out on the samples taken from different zones over the composite parts. Although the overall thickness of the composites produced by MAVIP in this study was found to be about $16 \%$ higher than the composites produced by CVIP, it was determined that incorporation of a membrane layer to vacuum infusion increases the fiber volume fraction while at the same time reducing the void content and thickness gradient across the part at no expense of compaction. At the vacuum port, strength and modulus values of the composites produced by MAVIP were found to be, respectively, 11 and $8 \%$ higher than those of the composites produced by CVIP. On the other hand, at the inlet port, composites produced by MAVIP exhibited 12 and $9 \%$ high strength and modulus values compared to those produced by CVIP. The load-deflection response $(\mathrm{P} / \delta)$ as a function of the length-to-width aspect ratio $(\mathrm{L} / \mathrm{b})$ was also experimentally determined. Composites produced by MAVIP were investigated to show the highest plot slope anywhere taken from the selected zones over the parts, irrespective of the ports. The findings were fitted to plane 
strain approach that was found to provide the lower bound for the beam modulus values. Once $\mathrm{L} / \mathrm{b}$ aspect ratio is greater than 6.5 , the plane strain approach seems to be converged to more definite beam modulus, regardless of the process used. In conclusion, incorporation of a membrane layer into vacuum infusion enhanced the dimensional stability, thus holding promise for more homogenous mechanical property variation combined with a relatively low void content distribution across the composite part.

\section{ACKNOWLEDGMENT}

The authors would cordially like to thank Anadolu University, Turkey, and Trans-Textil GmbH, Germany, for funding this project, kindly deeming it the first small step to encourage a reliable partnership in a long term in the near future.

\section{REFERENCES}

[1] Tanoğlu M, Seyhan AT. Investigating the effects of a polyester performing binder on the mechanical and ballistic performance of E-glass fiber reinforced polyester composites. Int. J. Adhes Adhes. 2003; $23: 1-8$

[2] Seyhan AT. A statistical study of fatigue life prediction of fibre reinforced polymer composites. Polym Polym Compos. 2011; 19:717- 723

[3] Li W, Krehl J, Gillespie JW, Heider D, Endrulat M, Hochrein K, Dunham MG, Dubois CB. Process and performance evaluation of the vacuum assisted process. J. Compo Mat. 2004; 38: 1803-1814

[4] Amouroux S, Heider D, Gillespie JW. Characterization of membranes used in pressure driven composite processing. Compos Part A-Appl S. 2010; 41:207-214

[5] Simacek P, Advani SG. Modeling resin flow and fiber tow saturation induced by distribution media collapse in VARTM. Compos. Sci. Technol. 2007; 67:2757-2769

[6] Acheson JA, Simacek P, Advani SG. The implications of fiber compaction and saturation on fully coupled VARTM simulation. Compos Part A-Appl S. 2004; 54:159-169

[7] Olivier P, Cottu J, Ferret B. Effects of cure cycle pressure and voids on some mechanical properties of carbon/epoxy laminates. Compos Part A-Appl S.2009; 95:509-515

[8] Harper BD, Staab GH, Chen RC. A Note on the Effects of Voids Upon the Hygral and Mechanical properties of AS4/3502 Graphite/Epoxy. J. composite material, 1987; 27:280-289

[9] M.C. Yu, S. Middleman. Air entrapment during liquid infiltration of porous media Chem. Eng. 1993; 123:61-69

[10] Kurematsu K, Koshi M. Kinetic studies on void formation during liquid epoxy resin impregnation through polyester nonwoven fabric. Colloid. Poly. Sci. 1985; 263:454-461

[11] Judd NCW, Wright WW. Voids and their effects on the mechanical properties of composites--an appraisal, Sampe J. 1978; 14:10-15

[12] Ghiorse SR, Jurta RM, Effects of low frequency vibration processing on carbon/epoxy laminates. Compos. $1991 ; 22: 3-8$ 
Seyhan / Anadolu Univ. J. of Sci. and Technology A-Appl. Sci. and Eng. 18 (2) - 2017

[13] Costa M, Almeida S, Cerqueira M. The influence of porosity on the interlaminar shear strength of carbon/epoxy and carbon/bismaleimide fabric laminates. Compos. Sci. Technol. 2001; 61:2101-2111

[14] Wood JR, Bader MG. Void control for polymer matrix composite (1): theoretical and experimental methods for determining the growth and collapse of gas bubbles. Compos Manuf. 1994; 4:224- 232

[15] Barbero E, Anido RL, Davalos F. On the Mechanics of Thin-Walled Laminated" Composite Beams. Compos Part A-Appl .1993; 27:806-817

[16] Bert CW. Simplified Analysis of Static Shear Factors for Beams of Non Homogeneous Cross Section. J. Compo Mat.,1973; 7:1925-1936

[17] Anido RL, Davalos F, Barbero E. Experimental evaluation of stiffness of laminated composite beam elements under flexural. J. Compo Mat.,1995; 14:49-61

[18] Rydarowski H, Koziol M. Repeatability of glass fiber reinforced polymer laminate panels manufactured by hand lay-up and vacuum-assisted resin infusion, J. Compo Mat 2015; 49:5-11

[19] Koziol M, Bator M, Rydarowski H, Hekner B. Comparative evaluation of GFRP laminate panels manufactured by VARI and RTM methods, Compos. Theo. and Prac., 2015; 15:196-207 